Morales-Ramírez, A., Till Pons, I., Alfaro, E. J., CorralesUgalde, M., \& Sheridan-Rodríguez, C. (2021). Respuestas del mesozooplancton a condiciones oceanográficas en diferentes escalas en Bahía Salinas, Pacífico Norte de Costa Rica, durante 2011-2013. Revista de Biología Tropical, 69(Suppl. 2), S142-S159. https://doi.org/10.15517/rbt. v69iS2.48741

\title{
Respuestas del mesozooplancton a condiciones oceanográficas en diferentes escalas en Bahía Salinas, Pacífico Norte de Costa Rica, durante 2011-2013
}

Álvaro Morales-Ramírez ${ }^{1,2}$; (D) https://orcid.org/0000-0002-0883-5366
Ivana Till Pons 3 ; (D) https://orcid.org/0000-0002-5057-2053
Eric J. Alfaro $1,4,5$; (D https://orcid.org/0000-0001-9278-5017
Marco Corrales-Ugalde ${ }^{6}$; (D https://orcid.org/0000-0001-5387-4914
Carolina Sheridan-Rodríguez ${ }^{2}$; (D) https://orcid.org/0000-0001-5881-8057

1. Centro de Investigación en Ciencias del Mar y Limnología (CIMAR), Universidad de Costa Rica, San José, Costa Rica; alvaro.morales@ucr.ac.cr

2. Escuela de Biología, Universidad de Costa Rica, San José, Costa Rica; carolinasheridan@gmail.com

3. Facultad de Ciencias Naturales, Universidad de la República, Uruguay; itill@fcien.edu.uy

4. Centro de Investigaciones Geofísicas, Universidad de Costa Rica, San José, Costa Rica

5. Escuela de Física, Universidad de Costa Rica, San José, Costa Rica; erick.alfaro@ucr.ac.cr

6. Oregon Institute of Marine Biology, University of Oregon, Eugene, USA; mcugalde88@gmail.com

Recibido 30-I-2021. Corregido 30-VI-2021. Aceptado 10-IX-2021.

\author{
ABSTRACT \\ Mesozooplankton responses to oceanographic conditions across different scales in Salinas Bay, \\ Northern Pacific coast of Costa Rica during 2011-2013
}

Introduction: The North Pacific of Costa Rica is characterized by presenting a variation of the subsurface temperature of the sea (SSST) modulated by surface winds with east component, with seasonal and intra-seasonal variations. The SSST is fundamental for the interactions of the ocean-atmosphere interface and influence marine biological processes. Zooplankton studies in the North Pacific are scarce and have been focused on the composition, abundance and biomass of macro and mesozooplankton in Culebra Bay. No works on zooplankton has been carried out northward of Papagayo Gulf.

Objective: To analyze the variation of the zooplankton in Bahía Salinas at different scales in response to oceanographic-atmospheric conditions.

Methods: during 2011, 2012 and 2013, mesozooplankton was collected in seven stations following a coastalocean gradient to determine abundance, biomass, and community composition. CTD casts were also carried out in each station. Hourly data of the Sea Subsurface Temperature (SSST) were obtained from June 2003 to December 2017.

Results: The annual surface temperature in Bahía Salinas was lower in December-April with a secondary minimum in July and higher in May-June and August-November. The cold, neutral and warm events determined by anomalies in the SSST, presented a distribution of the temperature in the water column with horizontal stratification, vertical mixture and homogeneous, respectively. The spatial distribution of zooplankton did not show significant differences and the variation of the total average abundance and biomass showed a similar behavior during the study period, with less variation in the first year compared to the second one, being the copepods the predominant category for all the dates. On a seasonal scale, a general pattern of variation between dry and rainy seasons was not observed, and copepods and others zooplankton groups were the categories that presented differences. On an intra-seasonal scale, abundance and biomass showed an inverse relationship with SST. Copepods and gelatinous zooplankton (GZ) were differentiated in all events. 
Conclusions: The zooplankton of Bahía Salinas respond differentially at different scales to the climatic conditions that affect the SSST of the region. The classification of the sampling dates into events allows characterizing different profiles in the water column it also allows to define the variation patterns for mesozooplankton that reflects short-term adaptation as a function of variation in environmental conditions. These findings help to understand how oceanographic processes determine plankton community composition and biota in general. This is relevant in times of climate change and the manifestation of its impact through processes such as ocean acidification and loss of marine biodiversity.

Key words: upwelling; subsurface temperature of the sea; Eastern Tropical Pacific; biomass; abundance; composition.

La región del Pacífico Norte de Costa Rica, conformada por el Golfo de Papagayo, el Golfo de Santa Elena y Bahía Salinas, se caracteriza por presentar una variación de la temperatura superficial del mar (TSM) modulada por patrones de vientos superficiales de componente este. Los principales vientos generadores de variabilidad son los vientos Alisios cuya velocidad varía a escala estacional, siendo mayores durante el invierno boreal y menores durante el verano boreal (Amador et al., 2016). La Corriente en Chorro de Bajo Nivel del Caribe (CLLJ por sus siglas en inglés) que ocurre en el invierno boreal, con uno de sus máximos en febrero, refuerza los vientos alisios (Amador, 2008), y en conjunto, causan una disminución en la TSM cuando los vientos son mayores (Alfaro \& Cortés, 2012; Alfaro \& Cortés, 2021; Alfaro et al., 2012; EscotoMurillo \& Alfaro, 2021; Fiedler \& Lavín, 2017). Este patrón estacional puede presentar variación intra-estacional dado por anomalías, como eventos de enfriamiento (calentamiento) más intensos en una escala temporal menor a la mensual y se relacionan con un aumento (disminución) mayor (menor) al promedio de vientos de componente este y noreste (Alfaro \& Cortés, 2012; Jiménez, 2001).

Los fuertes vientos del invierno boreal atraviesan los sistemas montañosos y producen un enfriamiento superficial por transferencia de calor latente debido a la evaporación y por un incremento en la capa de mezcla. Estos vientos fuertes además desplazan la capa superficial del agua de la costa hacia el oeste, produciendo surgencias de aguas más profundas, ricas en nutrientes, provocando los afloramientos costeros estacionales (Amador et al., 2016). Así, la variabilidad climática del Pacifico Tropical del Este (PTE) es determinada por procesos en la interfase océano-atmosfera, donde la TSM es fundamental para las interacciones de esta interfase y de gran influencia en los procesos biológicos marinos.

Diversos estudios han demostrado que la composición del zooplancton varía estacionalmente con los cambios físicos-químicos del ambiente (Bednarski \& Morales-Ramírez, 2004; Quesada-Alpízar \& Morales-Ramírez, 2004; Quesada-Alpízar \& Morales-Ramírez, 2006; Rodríguez-Saénz \& Morales-Ramírez, 2012) observándose modulaciones en la estructura de comunidad, las relaciones tróficas, los cambios fisiológicos y poblacionales (Bathman et al., 2001; Paffenhöfer, Hundley, \& Davis, 1989) así como patrones de distribución heterogénea en escalas espaciotemporales interdependientes de gradientes y procesos ambientales (Barry \& Dayton, 1991; Haury et al. 1978; Legendre \& Demers 1984; Pinel-Alloul, 1995; Steele, 1978). Los efectos de estas escalas se pueden cuantificar a partir de la presencia de los organismos, en sincronía con los eventos fisicoquímicos (Bednarski \& Morales-Ramírez, 2004; Quesada-Alpízar \& Morales-Ramírez, 2004; Quesada-Alpízar \& Morales-Ramírez, 2006; Rodríguez-Saénz \& Morales-Ramírez, 2012; Telesh \& Khlebovich, 2010).

Los estudios de zooplancton en el Pacífico Norte de Costa Rica son escasos. Para Bahía Culebra, en el Golfo de Papagayo, las investigaciones se han centrado en la composición, abundancia y biomasa del mesozooplacton y macrozooplancton marino, con énfasis en copépodos y medusas. Rodríguez-Sáenz y MoralesRamírez (2012) reportaron los patrones anuales 
de la composición, abundancia y distribución de mesozooplacton durante 1999-2000 y Bednarski y Morales-Ramírez (2004) estudiaron el macrozooplancton del año 2000 realizando comparaciones a escala estacional. Por otra parte, Morales-Ramírez et al. (2018) resaltan a Bahía Salinas como uno de los sitios de mayor diversidad del zooplancton en la zona costera del Pacífico Norte y de mayor abundancia registrada en el Pacífico costarricense.

El objetivo de este trabajo es analizar cuantitativamente la variación del zooplancton (abundancia, biomasa y composición general) de Bahía Salinas durante los años 2011-2013 a diferentes escalas como respuesta a condiciones oceanográficas-atmosféricas asociadas a la temperatura subsuperficial del mar (TSSM). Se plantea la hipótesis de trabajo de que dichos cambios son producto de la variabilidad de diversos procesos y eventos oceanográficosatmosféricos que se dan en el Pacífico Norte de Costa Rica.

\section{MATERIALES Y MÉTODOS}

Sitio de estudio: Bahía Salinas se encuentra en la parte más norte de Costa Rica (Fig. 1) sobre la vertiente tropical este del Océano Pacífico en el límite con Nicaragua. Esta bahía tiene un régimen climático con una marcada estación seca (diciembre a abril), un primer pico de la estación lluviosa (mayo y junio) y un segundo pico, mayor que el primero (setiembre y octubre); las lluvias disminuyen en julio y agosto en un período conocido localmente como veranillo de San Juan o canícula (Alfaro, 2002; Alfaro, 2014).

\section{Variables fisicoquímicas y biológicas:} Este estudio se realizó a partir de datos fisicoquímicos y biológicos obtenidos en siete estaciones de muestreo ubicadas en un gradiente costero-oceánico sobre la latitud 11.0057 y entre la longitud -85.6833 y -85.7917 , durante agosto del 2011 a agosto del 2013 (Fig. 1,

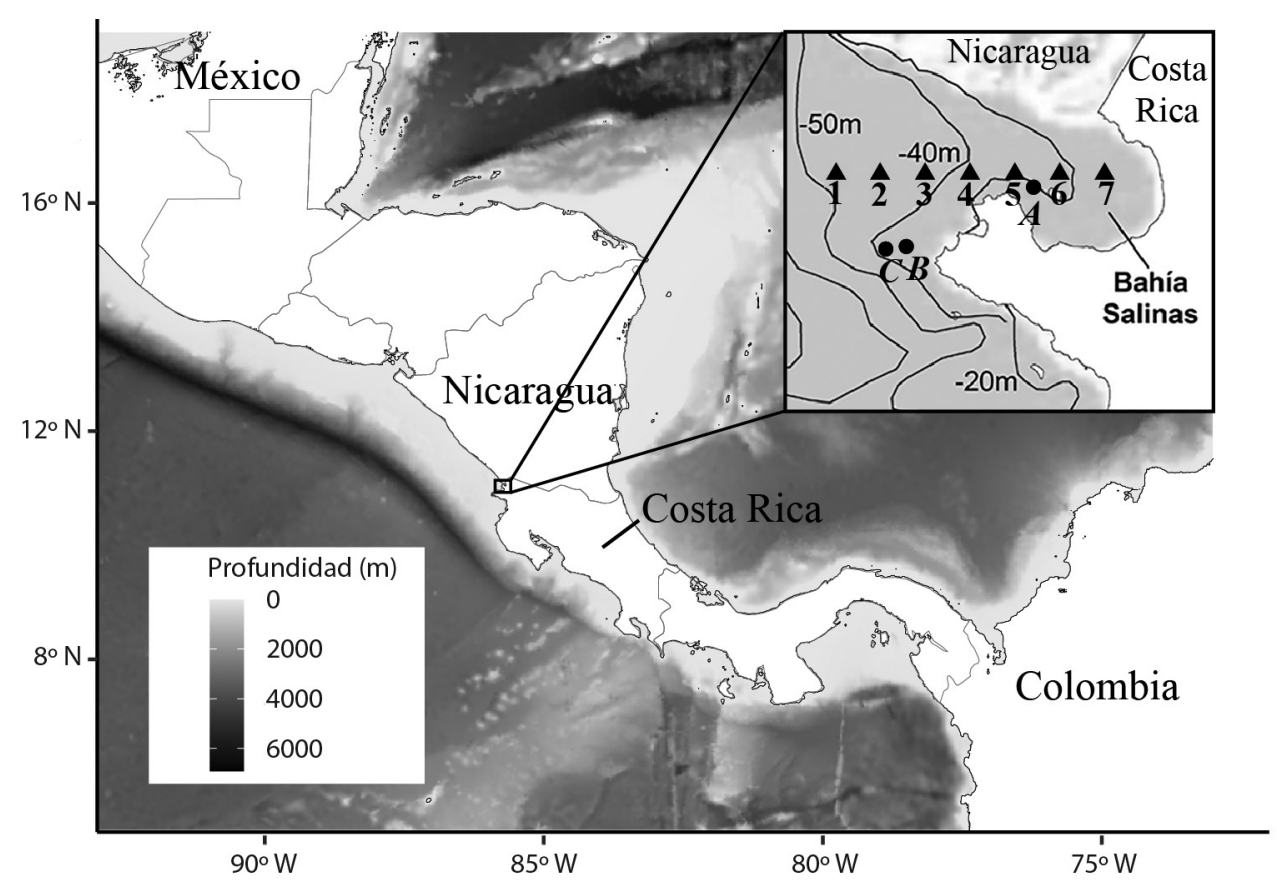

Fig. 1. Estaciones de muestreo de zooplancton y lances de CTD (triángulos) y puntos de medición de temperatura subsuperficial (círculos) en Bahía Salinas, Pacífico Norte de Costa Rica. 2011-2013.

Fig. 1. Zooplankton and CTD sampling stations (triangles), and subsurface temperature measurement stations (HOBO, circles), in Salinas Bay, North Pacific of Costa Rica, during 2011-2013. 
Tabla 1A). Además, se obtuvieron datos horarios de TSSM en siete estaciones cercanas a las anteriores utilizando sensores Hobotemp ${ }^{\circledR}$ (Onset Computer Corp) desde el 19 de junio de 2003 al 5 de diciembre de 2017 (Fig. 1, Tabla 1).

Durante los meses de agosto y diciembre del 2011, febrero, junio, agosto, octubre y diciembre 2012, y febrero, abril, junio y agosto del 2013, se tomaron muestras de mesozooplancton a partir de arrastres verticales desde la base de la termoclina con una red de $200 \mu \mathrm{m}$ de poro (ámbito de volumen filtrado 0.23-1.848 $\mathrm{m}^{-3}, 5-40 \mathrm{~m}$ profundidad). Las muestras fueron fijadas en formaldehido al $4 \%$ y fraccionadas con separador de plancton Folsom. Se tomó no más de una cuarta parte de la muestra para la determinación de biomasa seca según Postal, Fockland y Hagen (2000), el resto de la muestra se preservó en etanol al 70\%. Del resto de la muestra preservada en etanol, se tomaron alícuotas de $5 \mathrm{ml}$ con una pipeta Stempel para cuantificar y determinar la composición, hasta llegar a un conteo $>400$ organismos. Las identificaciones se realizaron según Boltovskoy (1999), Campos y Suárez-Morales (1994), Gasca y Suárez-Morales (1996) y PalomaresGarcía, Suárez-Morales y Hernández-Trujillo (1998). Se realizaron lances de CTD modelo SBE 19plus V2 en cada estación de muestreo para obtener datos de salinidad y temperatura de la columna de agua.

Análisis de datos: Las series de datos de TSSM se normalizaron para hacer sus registros comparables, restándole el promedio mensual histórico y dividiendo estas anomalías por su desviación estándar mensual histórica dado que se tomaron en distintas posiciones y profundidades (Tabla 1). Posteriormente los datos normalizados se rellenaron en cada una de las estaciones, ya que las mismas presentaban datos faltantes, siguiendo la metodología propuesta por Ureña, Alfaro y Soley (2016), que combina modelos autorregresivos y componentes principales. Se aplicó una media móvil triangular de 169 datos según lo propuesto por Soley (1994), para filtrar señales de alta

TABLA 1

Posición y profundidad de las estaciones de muestreo de zooplancton y lances de CTD (2011-2013) y ubicación, ámbito de profundidad y periodo de medición de las estaciones de Temperatura Subsuperficial del Mar en Bahía Salinas, Pacífico Norte de Cota Rica

TABLE 1

Location and depth of zooplankton sampling stations and CTD casts (2011-2013) and position, depth and measurement period of the stations for measuring the Subsurface Temperature of the Sea, Bahía Salinas, North Pacific of Costa Rica

\begin{tabular}{|c|c|c|c|c|c|c|}
\hline \multirow[b]{2}{*}{ Estación } & \multicolumn{2}{|c|}{ Zooplancton / CTD } & \multicolumn{4}{|c|}{ Medición de Temperatura Subsuperficial del Mar (TSSM) } \\
\hline & Coordenadas & $\begin{array}{l}\text { Profundidad } \\
\text { (m) }\end{array}$ & $\begin{array}{l}\text { Punto de } \\
\text { medición }\end{array}$ & Coordenadas & $\begin{array}{c}\text { Ámbito de } \\
\text { profundidad (m) }\end{array}$ & $\begin{array}{c}\text { Periodo de } \\
\text { medición }\end{array}$ \\
\hline Salinas 1 & $11.0557^{\circ} \mathrm{N} ;-85.7917^{\circ} \mathrm{W}$ & 40 & $\mathrm{~A}$ & $11.0504^{\circ} \mathrm{N} ;-85.7120^{\circ} \mathrm{W}$ & $4.6-6$ & $\begin{array}{l}15 / 12 / 2003 \\
05 / 12 / 2017\end{array}$ \\
\hline Salinas 2 & $11.0557^{\circ} \mathrm{N} ;-85.7739^{\circ} \mathrm{W}$ & 40 & & & $6.7-8.1$ & $\begin{array}{l}19 / 06 / 2003 \\
05 / 12 / 2017\end{array}$ \\
\hline Salinas 3 & $11.0557^{\circ} \mathrm{N} ;-85.7558^{\circ} \mathrm{W}$ & 30 & B & $11.0269^{\circ} \mathrm{N} ;-85.7634^{\circ} \mathrm{W}$ & $3.3-4.9$ & $\begin{array}{l}23 / 08 / 2003 \\
05 / 12 / 2017\end{array}$ \\
\hline Salinas 4 & $11.0557^{\circ} \mathrm{N} ;-85.7377^{\circ} \mathrm{W}$ & 20 & & & $7.4-9.1$ & $\begin{array}{l}23 / 08 / 2003 \\
27 / 09 / 2016\end{array}$ \\
\hline Salinas 5 & $11.0557^{\circ} \mathrm{N} ;-85.7196^{\circ} \mathrm{W}$ & 15 & $\mathrm{C}$ & $11.0259^{\circ} \mathrm{N} ;-85.7716^{\circ} \mathrm{W}$ & $10.5-11.9$ & $\begin{array}{l}08 / 10 / 2003 \\
05 / 12 / 2017\end{array}$ \\
\hline Salinas 6 & $11.0557^{\circ} \mathrm{N} ;-85.7014^{\circ} \mathrm{W}$ & 12 & & & $19.7-20.9$ & $\begin{array}{l}16 / 12 / 2003 \\
05 / 12 / 2017\end{array}$ \\
\hline Salinas 7 & $11.0057^{\circ} \mathrm{N} ;-85.6833^{\circ} \mathrm{W}$ & 5 & & & $4.5-6.6$ & $\begin{array}{l}18 / 02 / 2004 \\
22 / 04 / 2009\end{array}$ \\
\hline
\end{tabular}


frecuencia y se promediaron los datos horarios de las siete estaciones ya rellenadas igual a lo realizado por Alfaro y Cortés (2012). Se calculó un Índice de Temperatura Subsuperficial del Mar (ITSSM) como el promedio aritmético de los siete registros de la TSSM normalizados. El ITSSM generado se utilizó para clasificar las fechas de los muestreos en eventos fríos, neutros y cálidos si los valores se encontraban por debajo del percentil 33, entre el percentil 33 y 67 , o por encima del percentil 67 , respectivamente. Un valor negativo (positivo) indica que la TSSM es menor (mayor) a la media histórica y el valor en sí mismo es una relación con la desviación estándar mensual histórica. Por otro lado, se caracterizó el perfil vertical de la temperatura promedio y salinidad promedio para dichos eventos y para la transición de eventos fríos a neutros, y de neutros a cálidos (considerando la transición como la diferencia de los valores de neutros - fríos y cálidos neutros) a partir del promedio de cada metro de profundidad de los lances de CTD en cada estación de muestreo.

Para el mesozooplancton se realizó un análisis espacial según la distancia de la costa de las estaciones, y varios análisis temporales a diferentes escalas según la agrupación de las fechas de muestreo en: escala bianual (todo el período de muestreo), escala anual (de agosto a agosto), escala estacional (estación seca y lluviosa, y período de afloramiento estacional) y escala intra-estacional (eventos fríos, neutros y cálidos). Para todos los análisis de composición, el zooplancton fue dividido en copépodos, zooplancton gelatinoso, larvas de invertebrados y otros (anfípodos, ostrácodos, cladóceros, tecosomatos e ictioplancton) y en todos los casos se calculó un intervalo de confianza (IC) del 95\%.

Se aplicó la prueba de Shapiro-Wilk a los valores de abundancia por categoría, abundancia y biomasa total, para el estudio de normalidad y el resultado fue corroborado con prueba de Anderson- Darling. Para comparar los datos y determinar si presentaban diferencias significativas se utilizó la prueba de Kruskal-Wallis, $\mathrm{y}$ en los casos que presentaron diferencias significativas se realizaron comparaciones múltiples mediante la prueba de Mann-Whitney mediante el software Past 4.05.

\section{RESULTADOS}

Temperatura y salinidad en Bahía Salinas: La temperatura superficial media anual en Bahía Salinas fue de $25.3{ }^{\circ} \mathrm{C}$ (Fig. 2A). Las temperaturas más frías se observaron en diciembre-abril $\left(\leq 21^{\circ} \mathrm{C}\right.$ en febrero) y un mínimo secundario en julio. Se identificaron dos máximos, en mayo-junio y agosto-octubre con temperaturas superiores a $27{ }^{\circ} \mathrm{C}$ (superiores a $28{ }^{\circ} \mathrm{C}$ en setiembre-octubre). Marzo fue el mes que presentó mayor variabilidad de temperatura y la menor se observó en setiembre. El ITSSM varió a lo largo del período de estudio en el rango de -2.16 a 1.37 y los percentiles 33 y 67 calculados fueron -0.286 y 0.368 , respectivamente (Fig. 2B).

La Fig. 3 muestra el perfil de la temperatura y la salinidad promedio en el área de estudio. La amplitud de los rangos de temperatura promedio de la columna de agua disminuyeron de eventos fríos a eventos cálidos: $11.2{ }^{\circ} \mathrm{C}$ en eventos fríos (Fig. 3A), $9.9^{\circ} \mathrm{C}$ en eventos neutros (Fig. 3B) y $2.0^{\circ} \mathrm{C}$ en eventos cálidos (Fig. $3 \mathrm{C})$. En los eventos fríos la distribución de la temperatura presentó una estratificación horizontal, en los eventos neutros se observó un patrón de mezcla de variación principalmente vertical, y en los eventos cálidos una distribución general homogénea. La amplitud de los rangos de salinidad promedio de la columna de agua fueron: 0.69 en eventos fríos (Fig. 3D), 3.96 en eventos neutros (Fig. 3E) y 1.97 en eventos cálidos (Fig. 3F). La distribución de la salinidad en el perfil fue similar a la de la temperatura, coincidiendo las bajas salinidades con las altas temperaturas.

La transición de eventos fríos a neutros (Fig. 4A) tuvo variaciones menores de temperatura en comparación con la de neutros a cálidos a lo largo de la columna de agua (Fig. 4B) y presentó aumentos de hasta $8.8{ }^{\circ} \mathrm{C}$ y disminuciones de hasta $3.7^{\circ} \mathrm{C}$. La transición de eventos neutros a cálidos tuvo un aumento 
A

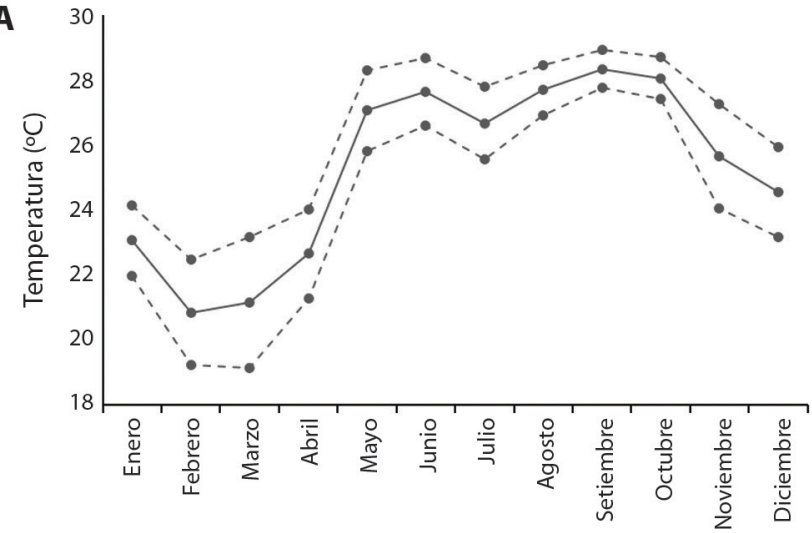

B

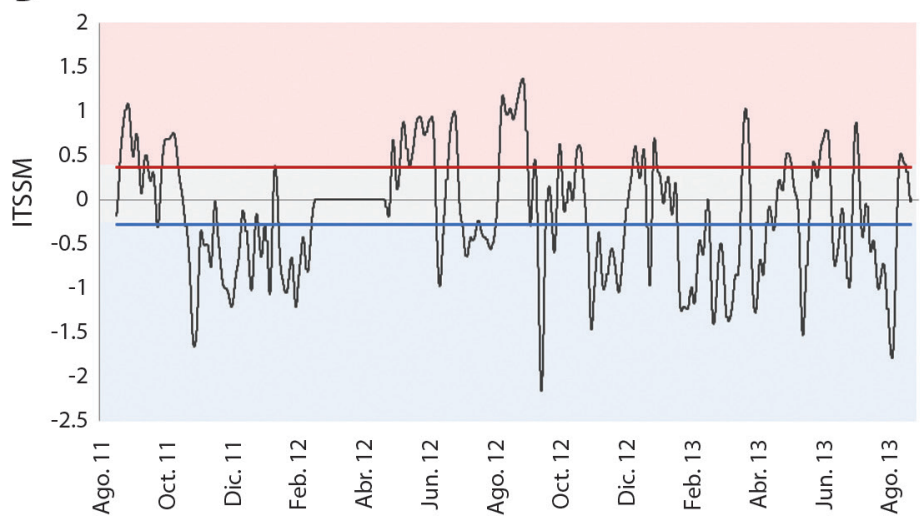

Fig. 2. A) Promedio mensual de los registros de temperatura media subsuperficial del mar (línea sólida) en la estación Salinas 2 (Tabla 1) \pm 1 desviación estándar mensual (líneas a trazos). B) Serie de tiempo del ITSS, percentil 33 (azul) y percentil 67 (rojo). Se colorea el fondo según las fechas correspondan a eventos fríos (azul), neutros (gris) o cálidos (rojo), observados en Bahía Salinas, Pacífico Norte de Costa Rica, 2011-2013.

Fig. 2. A) Monthly average of sea subsurface mean temperature (solid line) record at Salinas 2 station (Table 1) \pm 1 monthly standard deviation (dashed lines). B) ITSS time series, 33rd (blue) and 67th (red) percentiles. The background is colored according to the dates corresponding to cold (blue), neutral (grey) or warm (red) events observed in Salinas Bay, Costa Rica North Pacific, 2011-2013.

de temperatura generalizado (Fig. 4B). Mostró aumentos de hasta $10.5^{\circ} \mathrm{C}$ y disminuciones de hasta $0.1{ }^{\circ} \mathrm{C}$, por lo que podría generalizarse como un aumento en toda su extensión del perfil de la bahía.

La salinidad disminuyó para la transición de frío a neutro y de neutro a cálido (Fig. 4C, Fig. 4D), y fue mayor la diferencia para este último pasaje. Presentó disminuciones de hasta 1.4 para la primera transición y 2.6 para la segunda. En ambas se observó que donde más aumentó la temperatura, más disminuyó la salinidad y que la disminución fue prácticamente generalizada en todo el perfil a diferencia de la temperatura que presenta diferentes patrones.

Variación mesozooplancton en las diferentes escalas: El análisis espacial de la distribución del zooplancton no presentó diferencias significativas para la abundancia y biomasa promedio total (valor $\mathrm{p}>0.05$ ).

Escala bianual y anual: Los valores mínimos y máximos absolutos de abundancia (8281 ind $* \mathrm{~m}^{-3}$ y 205899 ind $\left.* \mathrm{~m}^{-3}\right)$ y biomasa (18.4 mg PS * $\mathrm{m}^{-3}$ y $86.2 \mathrm{mg}$ PS * $\mathrm{m}^{-3}$ ) fueron 

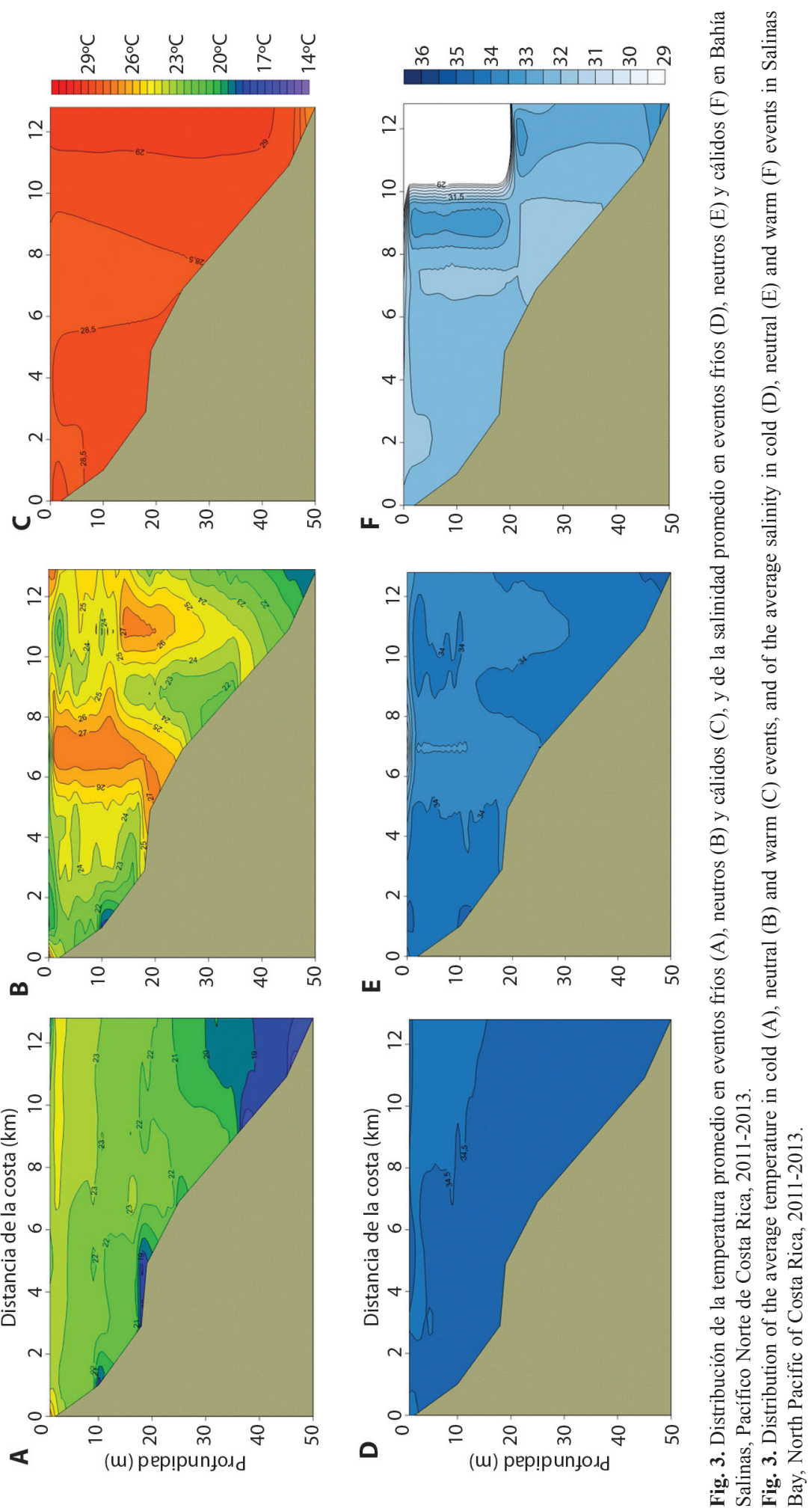
A Distancia de la costa $(\mathrm{km})$
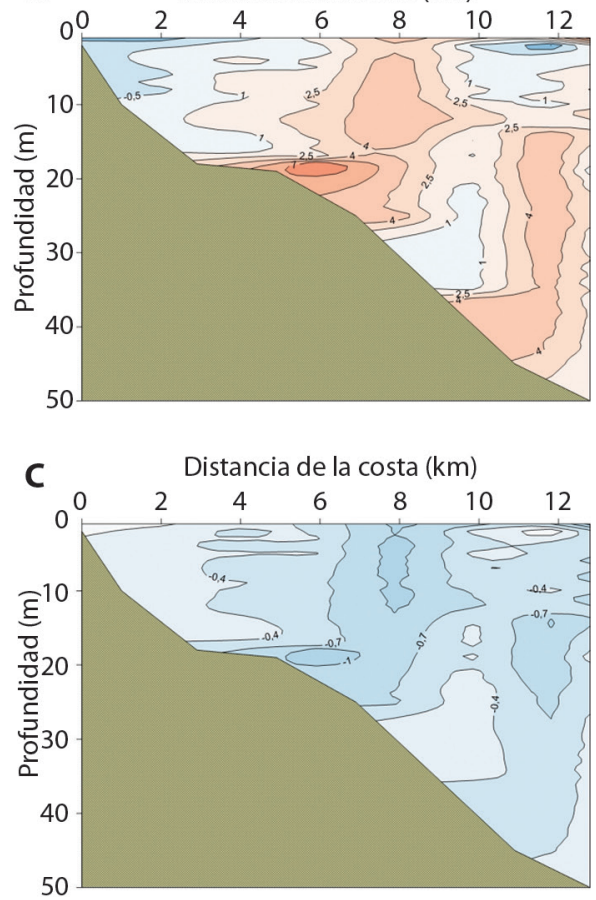

B

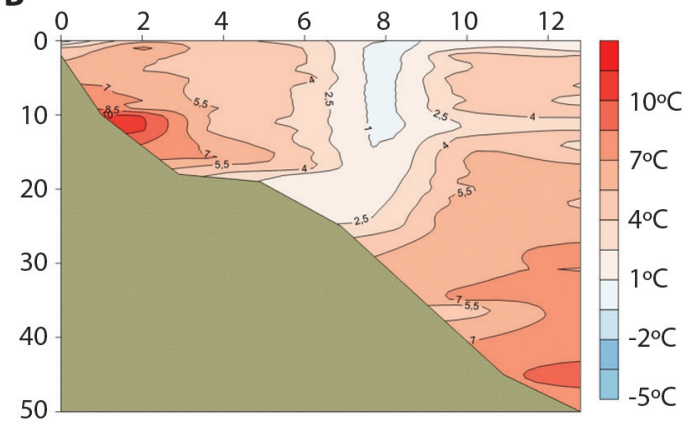

D

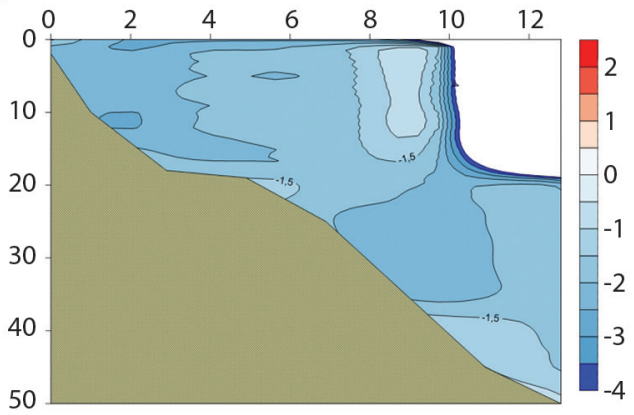

Fig. 4. Diferencia de temperatura promedio entre eventos neutros-fríos (A) y entre cálidos-neutros (B), y diferencia de salinidad promedio entre eventos neutros-fríos (C) y entre cálidos-neutros (D) en Bahía Salinas, Pacífico Norte de Costa Rica, 2011-2013.

Fig. 4. Average temperature difference between neutral-cold (A) and between warm-neutral (B) events and difference in average salinity between neutral-cold (C) and between warm-neutral (D) events in Salinas Bay, North Pacific of Costa Rica, 2011-2013.

significativamente diferentes entre si $(\mathrm{P}=0.002$ y $\mathrm{P}=0.002$, respectivamente), los mínimos se dieron en el bimestre agosto-octubre de 2012 y los máximos en febrero de 2013 (Fig. 5A). La abundancia y la biomasa promedio total presentaron menor variación en el primer año en relación al segundo (considerados de agosto a agosto del siguiente).

En el primer año de estudio los máximos de abundancia (73431 ind $* \mathrm{~m}^{-3}$ ) y biomasa $\left(52.1 \mathrm{mg}\right.$ PS $\left.* \mathrm{~m}^{-3}\right)$ promedio total fueron en junio de 2012 y febrero de 2012, respectivamente, y la abundancia (9297 ind * $\mathrm{m}^{-3}$ ) y biomasa $\left(36.0 \mathrm{mg}\right.$ PS $\left.* \mathrm{~m}^{-3}\right)$ mínima se dieron en agosto de 2011 para ambas variables. En este año el máximo de abundancia se da en la estación lluviosa. El máximo y mínimo de abundancia fueron significativamente diferentes $(\mathrm{P}=0.005)$ a diferencia de los valores de biomasa $(\mathrm{P}=0.055)$.

En el segundo año los máximos de ambas variables fueron en febrero de 2013 y los mínimos en el bimestre agosto-octubre de 2012. En este año los mínimos se dan en la estación lluviosa y los máximos durante la estación seca. Los máximos y mínimos fueron significativamente diferentes entre si para ambas variables $(\mathrm{P}=0.002$ para abundancia, $\mathrm{P}=0.002$ para biomasa), pero las transiciones de uno a otro fueron paulatinas.

El comportamiento de las abundancias de los diferentes grupos analizados fue similar al de la abundancia general (Fig. 5B). Los copépodos fueron predominantes en la abundancia total, para todas las fechas. Su abundancia máxima y mínima fueron significativamente 

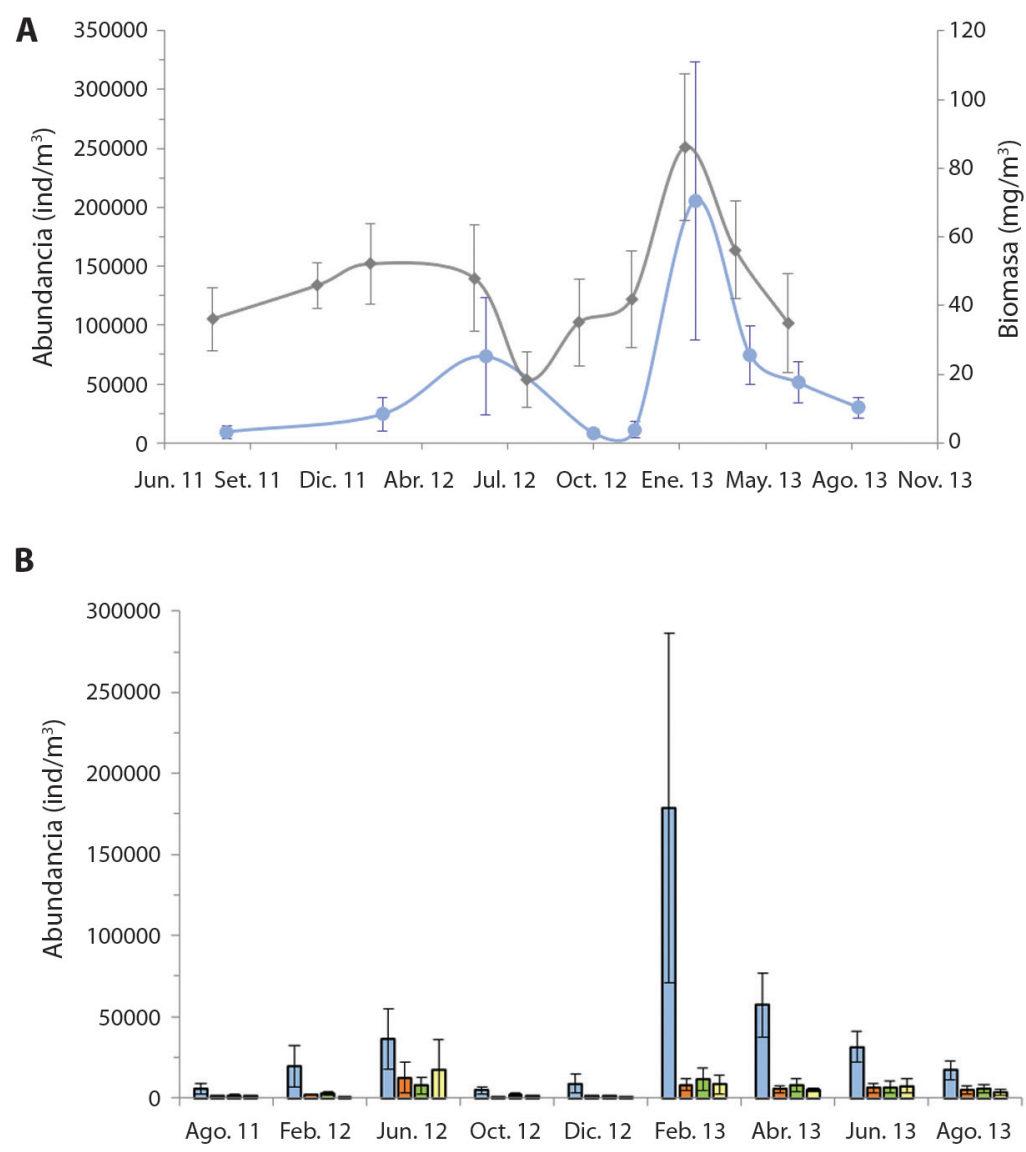

Fig. 5. A) Abundancia (celeste) y biomasa (gris) promedio total por fecha de muestreo. B) Abundancia promedio por fecha de muestreo de las categorías Copépodos (celeste), Zooplancton gelatinoso (naranja), Larvas (verde) y Otros (amarillo). Las barras corresponden al IC. Bahía Salinas, Pacífico Norte de Costa Rica. 2011-2013.

Fig. 5. A) Total average abundance (blue) and biomass (gray) by sampling date. B) Average abundance by sampling date of the categories Copepods (blue), Gelatinous Zooplankton (orange), Larvae (green) and Others (yellow). The bars correspond to the IC. Salinas Bay, North Pacific of Costa Rica. 2011-2013.

diferente entre sí $(\mathrm{P}=0.002)$ : la máxima fue en febrero de 2013 (178516 ind $* \mathrm{~m}^{-3}$ en la estación seca) y la mínima en octubre de 2012 (4591 ind * $\mathrm{m}^{-3}$ durante la estación lluviosa). Para el zooplancton gelatinoso $(\mathrm{ZG})$ el máximo fue en junio $2012\left(12316\right.$ ind $\left.* \mathrm{~m}^{-3}\right)$ y el mínimo en octubre 2012 (641 ind $\left.* \mathrm{~m}^{-3}\right)$, diferentes significativamente $(\mathrm{P}=0.002)$, ambos en la estación lluviosa. Las larvas tuvieron su mínimo en diciembre $2012\left(1051\right.$ ind $\left.* \mathrm{~m}^{-3}\right)$ y su máximo en febrero de 2013 (11160 ind * $\left.\mathrm{m}^{-3}\right)$, diferentes significativamente $(\mathrm{P}=0.01)$, ambos en estación seca. Para la categoría "otros" el mínimo fue en diciembre de 2012 (406 ind $* \mathrm{~m}^{-3}$ en estación seca) y el máximo en junio del 2012 (17448 ind $* \mathrm{~m}^{-3}$ en estación lluviosa) y fueron significativamente diferentes $(P=0.002)$.

Escala estacional: La variación de la abundancia no presentó un patrón general al clasificar las fechas en estación seca (diciembre-abril) y lluviosa (mayo-noviembre) (Fig. 6A). La abundancia promedio de la estación seca en los años 2012 y 2013 (97314.3 ind $* \mathrm{~m}^{-3}$ ) fue 3.3 veces mayor que la estación 
A

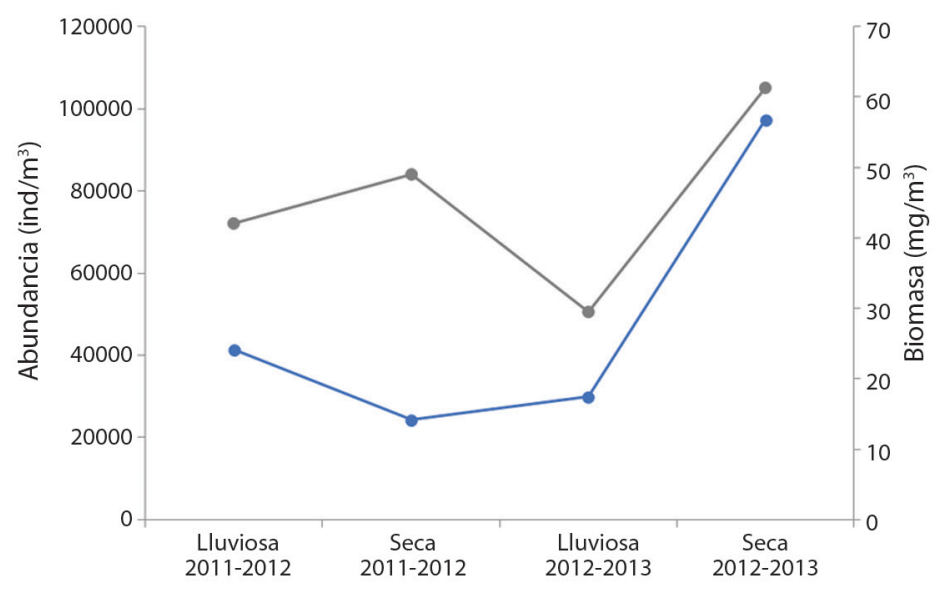

B

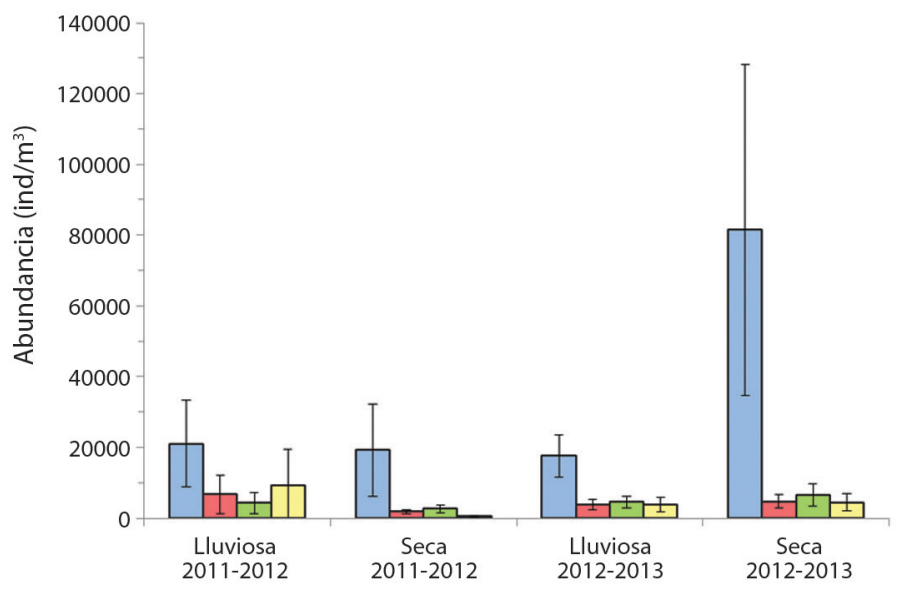

Fig. 6. A) Abundancia (celeste) y biomasa (gris) promedio total por estación. B) Abundancia promedio por estación. de las categorías Copépodos (celeste), Zooplancton gelatinoso (naranja), Larvas (verde) y Otros (amarillo). Las barras corresponden al IC, Bahía Salinas, Pacífico Norte de Costa Rica. 2011-2013.

Fig. 6. A) Total average abundance (blue) and biomass (gray) by season. B) Average abundance by station of the categories Copepods (blue), Gelatinous Zooplankton (orange), Larvae (green) and Others (yellow). The bars correspond to the CI. Salinas Bay, North Pacific of Costa Rica. 2011-2013.

lluviosa de esos años $\left(29846.8\right.$ ind $\left.* \mathrm{~m}^{-3}\right)$, siendo estas la única que se diferenció significativamente $(\mathrm{P}=0.04)$, al igual que los copépodos $(\mathrm{P}$ $=0.008)$ con mayor abundancia en la estación seca de los años 2012 y 2013 (81480 ind * $\left.\mathrm{m}^{-3}\right)$. Las restantes tres categorías presentaron las abundancias máximas en la estación lluviosa de los años 2011 y 2012, siendo otros la única con diferencias significativas entre seca y lluviosa, años 2011 y 2012 ( $\mathrm{P}=0.006)$, y por lo tanto la categoría que se ve afectada negativamente bajo las condiciones de la estación seca en los años 2011 y 2012 (Fig. 6B).

La biomasa aumentó en los pasos de estaciones lluviosas a secas y disminuyó de estaciones secas a lluviosas (Fig. 6A). Las estaciones seca y lluviosa de los años 2011 y 2012 no presentaron diferencias significativas $(\mathrm{P}=0.2)$, mientras que en el segundo año, las estaciones seca y lluviosa de los años 2012 y 2013 sí lo hicieron $(\mathrm{P}=0.0002)$. Las biomasas de las estaciones secas no se diferenciaron 

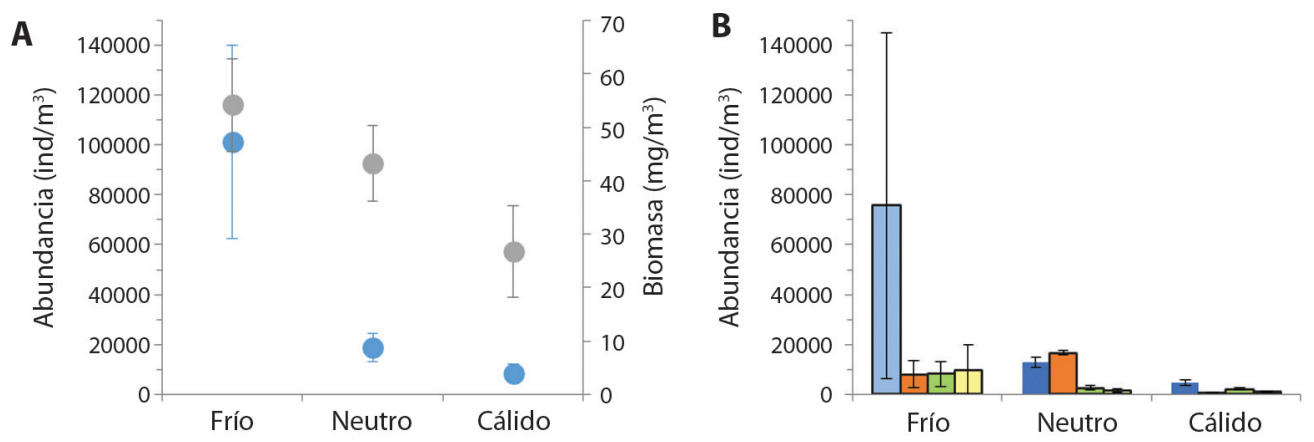

Fig. 7. A) Abundancia (celeste) y biomasa (gris) promedio total por eventos. B) Abundancias promedios de las categorías Copépoda (celeste), Zooplancton gelatinoso (naranja), Larvas (verde) y Otros (amarillo) por eventos. Las barras corresponden al IC. Bahía Salinas, Pacífico Norte de Costa Rica. 2011-2013.

Fig. 7. A) Total average abundance (blue) and biomass (gray) by events. B) Average abundances by events of the categories Copepod (blue), Gelatinous zooplankton (orange), Larvae (green) and Others (yellow). The bars correspond to the CI. Salinas Bay, North Pacific of Costa Rica. 2011-2013.

entre sí $(\mathrm{P}=0.3)$, siendo la estación seca años 2012 y 2013 la de mayor biomasa absoluta $\left(61.28 \mathrm{mg}^{*} \mathrm{~m}^{-3}\right)$ y presentó diferencias con las biomasas de las estaciones lluviosas ( $\mathrm{P}=0.03$, años 2011y 2012; y $\mathrm{P}=0.0002$, años 2012 y 2013). Las estaciones lluviosas se diferenciaron de manera significativa $(\mathrm{P}=0.03)$, siendo la lluviosa de los años 2011 y 2012 la de menor biomasa $\left(29.45 \mathrm{mg}\right.$ PS * $\left.\mathrm{m}^{-3}\right)$.

Escala intra-estacional: La abundancia y la biomasa total fueron mayores en eventos fríos y menores en eventos cálidos (Fig. 7A). La biomasa total presentó diferencias significativas entre eventos cálidos con los eventos neutros y fríos $(\mathrm{P}=0.009$ y $\mathrm{P}=0.0003$, respectivamente); la biomasa durante los eventos neutros y fríos no se diferenciaron entre sí. $\mathrm{La}$ abundancia total presentó diferencia durante los eventos fríos en comparación con los neutros y cálidos $(\mathrm{P}<0.001$ en ambos casos), sin diferenciar eventos neutros de cálidos.

Para los copépodos y ZG (los grupos dominantes), las abundancias se diferenciaron significativamente entre todos los eventos (frío y neutro: $\mathrm{P}<0.001$ en ambos, neutro y cálido: $\mathrm{P}=0.04$ y $\mathrm{P}=0.005$, respectivamente, cálido y frío: $\mathrm{P}<0.001$ para ambos casos) (Fig. 7B). La abundancia de copépodos alcanzó el 75\%, $38 \%$ y $55 \%$ de la abundancia promedio total en eventos fríos, neutros y cálidos respectivamente. Los eventos neutros favorecieron la presencia de zooplancton gelatinoso como grupo de abundancia predominante ya que alcanza el $8 \%, 50 \%$ y $8 \%$ de la abundancia promedio total en los eventos fríos, neutros y cálidos respectivamente.

Los grupos larvas y otros se diferenciaron significativamente solo en eventos fríos (frío y neutro $\mathrm{P}<0.001$ en ambos, frío y cálido $\mathrm{P}=$ 0.006 y $\mathrm{P}<0.001$, respectivamente), y presentaron una tendencia a disminuir la abundancia durante eventos fríos a cálido.

\section{DISCUSIÓN}

Temperatura subsuperficial del mar (TSSM): La variación anual de la temperatura subsuperficial del mar en Bahía Salinas se relaciona con el enfriamiento superficial, debido a la transferencia de calor latente por evaporación, el incremento de la capa de mezcla, el afloramiento estacional que caracteriza las costas del norte de Costa Rica durante el invierno boreal (Alfaro \& Cortés, 2012; Alfaro \& Cortés, 2021; Alfaro et al. 2012; Escoto-Murillo \& Alfaro, 2021) y el ciclo anual de la temperatura media de la superficie del aire en Bahía Culebra, Golfo de Papagayo (Alfaro et al. 2012). La TSSM es alta (mayo a noviembre) en los 
meses que no hay afloramiento y coincide con el debilitamiento de los vientos alisios sobre la región durante la estación lluviosa (Alfaro, 2002; Alfaro et al., 2018). La TSSM más fría (diciembre a abril) se observa en el periodo de afloramiento estacional, cuando los vientos alisios son más fuertes sobre la región y se presenta la estación seca (Alfaro et al., 2018). Un mínimo secundario en julio concuerda con el llamado veranillo de San Juan o Canícula (Alfaro, 2002; Alfaro, 2014) y con los máximos del CLLJ descritos por Amador (2008) que refuerzan los vientos de componente este. La relación inversa de la temperatura-salinidad encontrada en Bahía Salinas corresponde a la relación general que presentan éstas y es igual a lo observado por Bednarski y Morales-Ramírez (2004) en la región norte de Costa Rica (en Bahía Culebra). La clasificación de las fechas de muestreo en eventos, a partir del ITSSM, permitió identificar perfiles de la columna de agua con características diferentes para cada evento y patrones para la variación general del mesozooplancton.

Mesozooplancton: La variación se presenta en la escala temporal con una tendencia a aumentos de abundancia y biomasa al comienzo del período de afloramiento estacional durante la estación seca y disminuciones en el período de no afloramiento, durante la estación lluviosa. Algo similar a lo encontrado en la región norte por Bednarski y MoralesRamírez (2004), y por Rodríguez-Saénz y Morales-Ramírez (2012).

El rango de valores de las abundancias registrados para Bahía Salinas es mucho mayor al registrado hasta entonces en el norte de Costa Rica en Bahía Culebra para mesozooplancton (Rodríguez-Saénz \& Morales-Ramírez, 2012) y macrozooplancton (Bednarski \& MoralesRamírez, 2004), principalmente por presentar un máximo mucho mayor. Tanto Bahía Salinas como Bahía Culebra se ven afectadas por el mismo paso de vientos que genera los afloramientos estacionales como sucede en el golfo de Tehuantepec en México (Jiménez, 2001). En este lugar se encontraron diferencias de la biomasa y bioquímica del zooplancton en las diferentes regiones de la misma bahía ante un mismo evento frío de temperatura superficial del agua (Färber-Lorda et al., 2004). Proponemos que sería de utilidad realizar un estudio comparativo en simultáneo en las dos bahías (Salinas y Culebra) para explicar si la diferencia en el norte de Costa Rica se debe a que los vientos afectan las bahías de distinta manera, o si se relaciona a otras características como la influencia de aguas continentales (Bednarski \& Morales-Ramírez, 2004) o el efecto antrópico, ya que Bahía Culebra ha tenido un desarrollo turístico importante en su costa.

Mesozooplancton y TSSM: Los patrones de variación que presenta la estructura del mesozooplancton en función de la TSSM en las diferentes escalas de análisis pueden interpretarse por los fenómenos y eventos oceánicos-atmosféricos que actúan en las diferentes escalas. Alfaro y Lizano (2001) encontraron que el El Niño-Oscilación del Sur domina la variabilidad interanual de la TSM en las zonas de surgencia del PTE. El primer año de este estudio (de agosto 2011 a agosto 2012) el paso al período de afloramiento y el período de afloramiento sucedió bajo La Niña y no presentó diferencias significativas en la biomasa y abundancia que permitan establecer un patrón de variación del mesozooplancton. Desde agosto de 2011 hasta finales de abril del 2012 se estuvo bajo el fenómeno de La Niña (Poleo, 2011), que se disipó y dio paso a un evento de El Niño con un máximo en junio-julio 2012 (Poleo \& Stolz, 2012). En el segundo año (de agosto 2012 a agosto 2013) los cambios en la abundancia y biomasa presentaron diferencias significativas y se encontró un patrón de variación donde los valores mínimos se dan en período de no afloramiento y los máximos durante período de afloramiento. En este año el paso al período de afloramiento y el mismo afloramiento, sucedieron durante fase neutra de ENOS. El fenómeno de El Niño estuvo presente hasta octubre de 2012, y después pasó a fase neutral hasta agosto de 2013 (Naranjo, 2013). Se observa una tendencia a que la bioproductividad en Bahía 
Salinas se ve disminuida ante la presencia del fenómeno de La Niña y favorecida cuando el ENOS se encuentra en fase neutra. Estos resultados son diferentes a los encontrados por Rodríguez-Saénz y Morales-Ramírez (2012) en Bahía Culebra, donde las abundancias de la mayoría de los grupos de su estudio fueron mayores durante La Niña, lo que se corresponde a que este fenómeno afecte la estructura planctónica a esta escala, aunque todavía no se pueda determinar exactamente de qué manera.

En el análisis a escala estacional se esperaba encontrar diferencias entre el período de afloramiento y el de no afloramiento, pero se encontró diferencias significativas únicamente bajo las condiciones ambientales del segundo año de estudio y para el mesozooplancton y los copépodos. Los resultados, además, permiten observar que las condiciones ambientales de la estación seca, años 2011 y 2012, afectó negativamente a la categoría otros, contrario a lo que se esperaría ya que las estaciones secas favorecen la bioproductividad (Amador et al., 2016).

En el primer año los meses de la estación lluviosa no presentaron características normales, se destaca el mes de junio de 2012, que fue el tercer mes más seco del registro histórico, presentando un adelanto del "veranillo" (Naranjo, 2012). Esto explicaría el máximo de abundancia anual en un mes de no afloramiento y el gran aporte que genera a la abundancia total de los meses lluviosos explicando en parte, por qué la abundancia de la estación lluviosa es mayor a la seca en este año. Los meses de estación seca de 2011 sí presentaron características sinópticas normales (Morera, 2011; Solano, 2011); sin embargo, no aumentó la abundancia con el paso de estación lluviosa a estación seca. Por otro lado, la biomasa sí aumentó con el paso de estación lluviosa a seca, pero no de manera significativa, denotando una tendencia a un efecto diferencial de la biomasa y abundancia, en relación con las condiciones ambientales y que se observó mejor en el análisis a escala intra-estacional. Los resultados del año 2011 muestran que el impacto de las condiciones climáticas que actúan a escala mayor afectan la estructura estacional, por lo que definir el mesozooplancton a esta escala de manera aislada de otros fenómenos presentes a mayor escala, como ENOS, no sería una buena referencia, al menos en estudios de período corto, que podrían no considerar la variabilidad intra-estacional que sucede.

En el segundo año las características sinópticas fueron en general más secas de lo normal. Los meses de la estación lluviosa presentaron esta característica a excepción de octubre (Naranjo \& Poleo, 2012): agosto de 2012 presentó déficit de lluvias (Poleo \& Stolz, 2012); en abril del 2013 se extendió la transición de período seco a lluvioso, con vientos de componente este por encima de lo normal (Chinchilla, 2013); junio de 2013 presentó condiciones ventosas (Poleo \& Stolz, 2013); y agosto de 2013 presentó un segundo período canicular debido a la aceleración del CLLJ (Naranjo, 2013), lo que reforzó los vientos de componente este en la región norte de Costa Rica (Alfaro et al., 2012). Los meses de la estación seca de este año no presentaron grandes diferencias a las normales: diciembre 2012 no presentó frentes fríos, por lo que continuó con algunas características de estación lluviosa (Solano, 2012) retrasando un poco el comienzo de la estación seca y febrero de 2013 presentó características sinópticas normales (Morera, 2013). La extensión de la transición de la estación seca a lluviosa en abril 2013 y el veranillo sostenido en agosto 2013 podrían explicar los valores altos encontrados para este período de estudio y para los meses de estación lluviosa. Otra observación a gran escala es que la variación de las condiciones dentro del primer año y la estabilidad del segundo junto con las características de un año seco, podrían explicar las diferencias entre años, y notar una tendencia a que el análisis estacional puede presentar un patrón de variación definido si las condiciones climáticas son estables. Sin embargo, el análisis a escala intra-estacional permite encontrar un patrón de variación mejor definido. La TSSM presenta oscilaciones a escala intra-estacional. Los eventos fríos en el agua subsuperficial pueden durar días hasta semanas en el norte de Costa Rica (Alfaro \& Cortés, 
2012). La variabilidad del mesozooplancton a esta escala permite ver que la abundancia y biomasa tienen una relación inversa con la TSSM y se ven afectadas de manera diferencial. Al aumentar la temperatura del agua (paso de evento frío a neutro) el zooplancton se ve afectado negativamente, y se observa una adaptación en la que primero disminuye el número de individuos (la abundancia es significativamente diferente para eventos fríos, de neutros y cálidos) y una tendencia a disminuir la biomasa. Luego ante un mayor aumento de temperatura (paso de eventos neutros a cálidos) disminuye la biomasa (es diferente significativamente para eventos cálidos, de neutros y fríos) y se observa una tendencia a disminuir la abundancia. Al considerarse, que la transición de eventos fríos a neutros tiene una diferencia de temperatura menor que la transición de neutros a cálidos, se puede observar que la abundancia del plancton de Bahía Salinas es más sensible al cambio de temperatura que la biomasa. Esto refleja la adaptación a corto plazo del mesozooplancton a los cambios ambientales debido a su ciclo de vida corto, similar a las respuestas rápidas del zooplancton a eventos fríos de que observó Färber-Lorda et al. (2004) en el Golfo de Tehuantepec. Sería como una estrategia $\mathrm{k}$ y r a escala planctónica, como respuesta a una diferencia de temperatura intra-estacional. La colonización de un ambiente generalmente comienza con especies de estrategia $r$ y luego comienzan a aparecer las especies de estrategia k (Vanschoenwinkel et al., 2011). El ambiente a colonizar sería el generado por un evento frío, en el que las surgencias de aguas profundas aportan grandes cargas de nutrientes.

Los copépodos y ZG son grupos que reflejan diferencias entre los tres eventos y la abundancia del ZG se ve favorecida en la transición de eventos fríos a neutros, lo que puede sugerir algún tipo de sucesión de organismos como respuesta a los cambios a escala intraestacional, ya que dentro de estas categorías existen gran diversidad de estrategias reproductivas y alimenticias. Estos comportamientos se han observado en la abundancia y diversidad de zooplancton gelatinoso en el Golfo Dulce, Pacífico sur de Costa Rica (Morales-Ramírez \& Nowaczyk, 2006). Por esta razón, será útil profundizar en la composición de estos grupos, copépodos y ZG (Till-Pons, Morales-Ramírez, Corrales-Ugalde, Sheridan-Rodríguez, \& Alfaro, en prep.).

La información generada en este estudio es útil para estudios de mitigación de efectos antrópicos locales, así como para la toma de medidas de mitigación en el contexto de cambio climático que Alfaro et al. (2012) identifican desde la década de 1850, en la región Norte de Costa Rica. Estudios recientes han demostrado la influencia de la acidificación oceánica en esta región como una amenaza para los arrecifes y comunidades coralinas (Sánchez-Noguera et al., 2018), así como otros estudios lo han demostrado para el plancton marino (ÁlvarezFernández et al., 2018; Cripps, Flynn, \& Lindeque, 2016; Spilling et al., 2016, Taucher et al., 2017). Por lo tanto, es importante destacar específicamente la importancia de conectar los rasgos en poblaciones de zooplancton marino que son sensibles a la acidificación oceánica y cambios de temperatura, con aquellos que son ecológicamente relevantes y observables de manera confiable en el campo (Kein, Klinger, Keister, \& MacLaskey, 2021). Esto abre una serie de posibilidades para futuros estudios en este importante y moderno aspecto del cambio climático y su eventual impacto en la pérdida de diversidad marina, en la región del Pacífico Norte de nuestro país.

Declaración de ética: los autores declaran que todos están de acuerdo con esta publicación y que han hecho aportes que justifican su autoría; que no hay conflicto de interés de ningún tipo; y que han cumplido con todos los requisitos y procedimientos éticos y legales pertinentes. Todas las fuentes de financiamiento se detallan plena y claramente en la sección de agradecimientos. El respectivo documento legal firmado se encuentra en los archivos de la revista. 


\section{AGRADECIMIENTOS}

Se agradece a la OAICE y al SEP, UCR, y al programa de beca Iberoamericana, estudiante de grado Santander, por el apoyo económico brindado a Ivana Till durante su estadía en Costa Rica. A los centros de investigación CIMAR y CIGEFI de la UCR por su apoyo logístico durante la recopilación y análisis de datos. Esta es una contribución del proyecto 808-B1-194 (AMR), EA agradece a los proyectos: C0130, B9454, C0404, C0610, EC-497, C0074, que le dieron el tiempo y recursos para realizar esta investigación. Los autores agradecen a Jorge Cortés por facilitar los datos de CTD y de los hobos para realizar parte de la investigación a través del proyecto: Comunidades bentónicas y caracterización física y química de Bahía Salinas, Guanacaste (808-A5-037). También al señor Eléazar Ruiz, por su apoyo en el trabajo de campo. Agradecemos los comentarios y sugerencias de revisores externos.

\section{RESUMEN}

Introducción: El Pacífico Norte de Costa Rica se caracteriza por presentar una variación de la temperatura subsuperficial del mar (TSSM) modulada por vientos superficiales de componente este, con variaciones estacionales e intra-estacional. La TSSM es fundamental para las interacciones de la interfase océano-atmósfera y de gran influencia en los procesos biológicos marinos. Los estudios de zooplancton en el Pacífico Norte son escasos y se han enfocado en la composición, abundancia y biomasa de macro y mesozooplanctonen en Bahía Culebra. No se han realizado trabajos sobre zooplancton al norte del Golfo de Papagayo.

Objetivo: Analizar la variación del zooplancton de Bahía Salinas a diferentes escalas como respuesta a condiciones oceanográficas-atmosféricas.

Métodos: durante algunos meses de los años 2011, 2012 y 2013, el mesozooplancton fue muestreado en siete estaciones siguiendo un gradiente costero-oceánico para determinar su abundancia, biomasa y composición de la comunidad. Se realizaron lances de CTD en cada estación, y se recopilaron datos horarios de la TSSM desde junio de 2003 a diciembre de 2017.

Resultados: La temperatura superficial anual en Bahía Salinas es menor en diciembre-abril con un mínimo secundario en julio y mayor en mayo-junio, y agosto-octubre. Los eventos fríos, neutros y cálidos determinados por anomalías en la TSSM, presentaron una distribución de la temperatura en la columna de agua con estratificación horizontal, de mezcla vertical y homogénea, respectivamente. La distribución espacial del zooplancton no presentó diferencias significativas y la variación del promedio total de abundancia y biomasa mostró un comportamiento similar durante el período de estudio, con menor variación en el primer año en comparación con el segundo, siendo los copépodos la categoría predominante para todas las fechas. A escala estacional no se observó un patrón general de variación entre estación seca y lluviosa y, copépodos y otros grupos del zooplancton fueron las categorías que presentaron diferencias. A escala intra-estacional la abundancia y biomasa presentaron una relación inversa con la TSSM y se vieron afectadas de manera diferencial. Los copépodos y zooplancton gelatinoso se diferenciaron en todos los eventos.

Conclusiones: El zooplancton de Bahía de Salinas responde de manera diferencial en las diferentes escalas a las condiciones climáticas que afectan la TSSM de la región. La clasificación de las fechas de muestreo en eventos permite caracterizar diferentes perfiles en la columna de agua, así como permite definer patrones de variación para el mesozooplancton, que refleja la adaptación a corto plazo en función de la variación de las condiciones ambientales. El conocimiento generado ayuda a comprender mejor los fenómenos oceanográficos y su efecto sobre las poblaciones de plancton y la biota en general, especialmente en el escenario de cambio climático y las manifestaciones modernas de su impacto, p. e. la acidificación oceánica y pérdida de biodiversidad marina.

Palabras clave: afloramiento; temperatura subsuperficial del mar; Pacífico Tropical Oriental; biomasa; abundancia; composición.

\section{REFERENCIAS}

Alfaro, E. (2002). Some Characteristics of the Annual Precipitation Cycle in Central America and their Relationships with its Surrounding Tropical Oceans. Tópicos Meteorológicos y Oceanográficos, 9(2), 88-103.

Alfaro, E. (2014). Caracterización del "veranillo" en dos cuencas de la vertiente del Pacífico de Costa Rica, América Central. Revista de Biología Tropical, 62(Suplemento 4), S1-S15.

Alfaro, E., \& Cortés, J. (2012). Atmospheric forcing of cool subsurface water events in Bahía Culebra, Gulf of Papagayo, Costa Rica. Revista de Biología Tropical, 60(Supplement 2), S173-S186

Alfaro, E. J., \& Cortés, J. (2021). Forcing of cool and warm subsurface water events in Bahía Salinas, Costa Rica. Revista de Biologia Tropical, 69(Supplement 2), S127-S141.

Alfaro, E., Cortés, J., Alvarado, J. J., Jiménez, C., León, A., Sánchez-Noguera, C., Nivia-Ruiz, J., \& Ruiz, 
E. (2012). Clima y temperatura sub-superficial del mar en Bahía Culebra, Golfo de Papagayo, Costa Rica. Revista Biológica Tropical, 60(Suplemento 2), S159-S171.

Alfaro, E. J., Chourio, X., Muñoz, Á. G., \& Mason, S. J. (2018). Improved seasonal prediction skill of rainfall for the first season in Central America. International Journal of Climatology, 38(1), e255-e268.

Alfaro, E., \& Lizano, O. (2001). Algunas relaciones entre las zonas de surgencia del Pacífico Centroamericano y los Océanos Pacíficos y Atlántico Tropical. Revista de Biología Tropical, 49(Suplemento 2), S185-S193.

Álvarez-Fernández, S., Bachb, L.T., Taucherb, J., Riebesellb, U., Sommerb, U., Aberlec, N., Brussaardd, C. P. D., \& Boersma, M. (2018). Plankton responses to ocean acidification: The role of nutrient limitation. Progress in Oceanography, 165, 11-18.

Amador, J. A. (2008). The Intra-Americas Seas Low-Level Jet (IALLJ): Overview and Future Research. Annals of the New York Academy of Sciences, 1146(1), 153-188.

Amador, J. A., Durán-Quesada, M., Rivera, E. R., Mora, G., Sáenz, F., Calderón, B., \& Mora, N. (2016). The easternmost tropical Pacific. Part I: Seasonal and intraseasonal modes of atmospheric variability. Revista de Biología Tropical, 64(Supplement 1), S23-S57.

Barry, J. P., \& Dayton, P. K. (1991). Physical heterogeneity and the organization of marine communities. In Ecological heterogeneity (pp. 270-320). Springer, New York, NY.

Bathmann, U., Bundy, M. H., Clarke, M. E., Cowles, T. J., Daly, K., Dam, H. G., \& Wishner, K. F. (2001). Future marine zooplankton research - a perspective. Marine Zooplankton Colloquium 2. Marine Ecology Progress Series, 222, 297-308.

Bednarski, M., \& Morales-Ramírez, A. (2004). Composition, abundance and distribution of macrozooplankton in Culebra Bay, Gulf of Papagayo, Pacific coast of Costa Rica and its value as bioindicators of pollution. Revista de Biología Tropical, 53(Supplement 2), S105-S118.

Boltovstoy, S. (Ed.) (1999). South Atlantic Zooplankton. Vol. 1+2. Leiden, The Netherlands: Backhuys Publishers.

Campos, A., \& Suárez-Morales, E. (1994). Copépodos pelágicos del Golfo de México y Mar Caribe. I. Biología y Sistemática. Chetumal, México: CIQRO.

Chinchilla, G. (2013). Boletín Meterorológico Mensual, abril 2013. Boletín Meteorológico Mensual. Instituto Meteorológico de Costa Rica, San José, Costa Rica. https://www.imn.ac.cr/documents/10179/14637/ ABRIL
Cripps, G., Flynn, K. J., \& Lindeque, P. K. (2016). Ocean acidification affects the phyto-zooplankton trophic transfer efficiency. PLoS One, 11(4), e0151739. https://doi.org/10.1371/journal.pone.0151739

Escoto-Murillo, A., \& Alfaro, E. J. (2021). Análisis de eventos fríos y cálidos por medio de datos de buceo: un enfoque de ciencia ciudadana en el Golfo de Papagayo, Costa Rica. Revista de Biologia Tropical, 69(Supplement 2), S94-S104.

Färber-Lorda J., Lavín M. F., \& Guerrero-Ruiz, M. A. (2004). Effects of wind forcing on the trophic conditions, zooplankton biomass and krill biochemical composition in the Gulf of Tehuantepec. Deep-Sea Research II, 51, 601-614.

Fiedler, P. C., \& Lavín, M. F. (2017). Oceanographic conditions of the eastern tropical Pacific. In P. W. Glynn, D. Manzello \& I. Enochs (Eds.), Coral Reefs of the Eastern Pacific: Persistence and Loss in a Dynamic Environment (pp. 59-83). Dordrecht: Springer Science+Business Media.

Gasca, R., \& Suárez-Morales, E. (1996). Introduccion al estudio del zooplancton marino. Quintana Roo, México: ECOSUR/CONACIT.

Haury, L. R., McGowan, J. A., \& Wiebe, P. H. (1978). Patterns and processes in the time-space scales of plankton distributions. In J. H. Steele, Spatial Pattern in Plankton Communities (pp. 277-327). Boston: Springer.

Jiménez, C. (2001). Seawater temperature measured at the surface and at two depths $(7$ and $12 \mathrm{~m})$ in one coral reef at Culebra Bay, Gulf of Papagayo, Costa Rica. Revista de Biología Tropical, 49(Supplement 2), S153-S161.

Keil, K. E., Klinger, T. J, Keister, J. E., \& McLaskey, A. K. (2021). Comparative sensitivities of zooplankton to ocean acidification conditions in experimental and natural settings. Frontiers in Marine Sciences, 8 , 613778. https://doi.org/10.3389/fmars.2021.613778

Legendre, L., \& Demers, S. (1984). Towards dynamic biological oceanography and limnology. Canadian Journal of Fisheries and Aquatic Sciences, 41(1), 2-19.

Morales-Ramírez, A., Corrales-Ugalde, M., EsquivelGarrote, O., Carrillo-Baltodano, A., Rodríguez-Saénz, K, \& Sheridan, C. (2018). Estudios de zooplancton marino en Costa Rica: una revisión y perspectivas a futuro. Revista de Biología Tropical, 66(Suplemento 1), S24-S41.

Morales-Ramírez, A., \& Nowaczyk, J. (2006). El zooplancton gelatinoso del Golfo Dulce, Pacífico de Costa Rica, durante la transición de la estación lluviosa a la seca 1997-1998. Revista de Biología Tropical, 54(Suplemento 1), S201-S223. 
Morera, R. (2011). Boletín Meteorológico Mensual, febrero 2011. Boletín Meteorológico Mensual. Instituto Meteorológico de Costa Rica, San José, Costa Rica. https:/www.imn.ac.cr/documents/10179/14633/ FEBRERO

Morera, R. (2013). Boletín Meteorológico Mensual, febrero 2013. Boletín Meteorológico Mensual. Instituto Meteorológico de Costa Rica, San José, Costa Rica. https://www.imn.ac.cr/documents/10179/14637/ FEBRERO

Naranjo, J. (2012). Boletín Meteorológico Mensual, junio 2012. Boletín Meteorológico Mensual. Instituto Meteorológico de Costa Rica, San José, Costa Rica. https://www.imn.ac.cr/documents/10179/14635/ JUNIO

Naranjo, J. (2013). Boletín Meteorológico Mensual, agosto 2013. Boletín Meteorológico Mensual. Instituto Meteorológico de Costa Rica, San José, Costa Rica. https://www.imn.ac.cr/documents/10179/14637/ AGOSTO

Naranjo, J., \& Poleo, D. (2012). Boletín Meteorológico Mensual, octubre 2012. Boletín Meteorológico Mensual. Instituto Meteorológico de Costa Rica, San José, Costa Rica. https://www.imn.ac.cr/ documents/10179/14635/OCTUBRE

Paffenhöfer, G. A, Huntley, M. E., \& Davis, C. S. (1989). Future marine zooplankton research - a perspective. Marine Zooplankton Colloquium 1. Marine Ecology Progress Series, 55, 197-305.

Palomares-García, R., Suárez-Morales, E., \& HernándezTrujillo, S. (1998). Catálogo de los copépodos (Crustacea) pelágicos del Pacífico Mexicano. México: CICIMAR/ECOSUR.

Pinel-Alloul, P. (1995). Spatial heterogeneity as a multiscale characteristic of zooplankton community. Hydrobiologia, 300(1), 17-42.

Poleo, D. (2011). Boletín Meteorológico Mensual, abril 2011. Boletín Meteorológico Mensual. Instituto Meteorológico de Costa Rica, San José, Costa Rica. Tomado de https://www.imn.ac.cr/ documents/10179/14635/ABRIL

Poleo, D., \& Stolz, W. (2012). Boletín Meteorológico Mensual, agosto 2012. Boletín Meteorológico Mensual. Instituto Meteorológico de Costa Rica, San José, Costa Rica. https://www.imn.ac.cr/ documents/10179/14635/AGOSTO

Poleo, D., \& Stolz, W. (2013). Boletín Meteorológico Mensual, junio 2013. Boletín Meteorológico Mensual. Instituto Meteorológico de Costa Rica San José, Costa Rica. https://www.imn.ac.cr/ documents/10179/14637/JUNIO

Postal, L., Fockand, H., \& Hage, W. (2000). Abundance and biomass. In R. P. Harris, P. Wiebe, J. Lenz, H.
R. Skjoldal, \& M. Huntley (Eds.), ICES Zooplankton Methodology Manual (pp. 83-192). San Diego, California: Academic Press.

Quesada-Alpízar, M. A., \& Morales-Ramírez, A. (2004). Comportamiento de las masas de agua en el Golfo Dulce durante un período El Niño (1997-1998). Revista de Biología Tropical, 53(Suplemento 2), S95-S103.

Quesada-Alpízar, M. A., \& Morales-Ramírez, A. (2006). Abundancia, composición y distribución vertical del zooplancton no gelatinoso en el Golfo Dulce, costa pacífica de Costa Rica, durante 1997-1998: posible efecto fenómeno de El Niño. Revista de Biología Tropical, 54(Suplemento1), S225-S240.

Rodríguez-Saénz, K., \& Morales-Ramírez, A. (2012). Composición y distribución del mesozooplancton en una zona de afloramiento costero (Bahía Culebra, Pacífico Norte de Costa Rica) durante La Niña 1999 y el 2000. Revista de Biología Tropical, 60(Suplemento 2), S143-S157.

Sánchez-Noguera, C., Shuldreier, I., Cortés, J., MoralesRamírez, A., Wild, C., \& Rixen, T. (2018). Natural ocean acidification at Papagayo upwelling system (north Pacific Costa Rica): implications for reef development. Biogeosciences, 15, 2349-2360.

Solano, E. (2011). Boletín Meterorológico Mensual, diciembre 2011. Boletín Meteorológico Mensual. Instituto Meteorológico de Costa Rica, San José, Costa Rica. https://www.imn.ac.cr/documents/10179/14633/ DICIEMBRE

Solano, E. (2012). Boletín Meterorológico Mensual, diciembre 2012. Boletín Meteorológico Mensual. Instituto Meteorológico de Costa Rica, San José, Costa Rica. Tomado de https://www.imn.ac.cr/ documents/10179/14635/DICIEMBRE

Soley, F. J. (1994). Suavizamiento de series cronológicas geofísicas con ruido blanco y rojo aditivo. Revista de Geofisica, 41, 33-58.

Spilling, K., Allanah, J. P., Virkkala, N., Hastings, T., Lischka, S., Stuhr, A., Bermúdez, R., Czerny, J., Boxhammer, T., Schulz, K.G., Ludwig, A., \& Riebesell, U. (2016). Ocean acidification decreases plankton respiration: evidence from a mesocosm experiment. Biogeosciences, 13, 4707-4719.

Steele, J. H. (1978). Spatial Pattern in Plankton Communities (Vol. 3). Boston: Springer Science \& Business Media.

Taucher, J., Haunost, M., Boxhammer, T., Lennart, T. B., Algueró-Muñiz, M., \& Riebesell, U. (2017). Influence of ocean acidification on plankton community structure during a winter-to-summer succession: An imaging approach indicates that copepods can benefit from elevated $\mathrm{CO}_{2}$ via indirect food web effects. 
PLoS ONE, 12(2), e0169737. https://doi.org/10.1371/ journal.pone.0169737

Telesh, I. V., \& Khlebovich, V. V. (2010). Principal processes within the estuarine salinity gradient: a review. Marine Pollution Bulletin, 61(4-6), 149-155.

Ureña, P., Alfaro, E., \& Soley, J. (2016). Propuestas metodológicas para el rellenado de datos ausentes en series de tiempo geofísicas. Guía Práctica de uso. Documento Técnico. Centro de Investigaciones Geofísicas, Escuela de Física y
Centro de Investigaciones en Ciencias del Mar y Limnología, Universidad de Costa Rica. http://www. kerwa.ucr.ac.cr/handle/10669/28888.2

Vanschoenwinkel, B., Mergeay, J., Pinceel, T., Waterkeyn, A., Vandewaerde, H., Seaman, M., \& Brendonck, L. (2011). Long distance dispersal of zooplankton endemic to isolated mountaintops - an example of an ecological process operating on an evolutionary time scale. PLoS ONE, 6(11), e26730. https://doi. org/10.1371/journal.pone.0026730 